\title{
Field Orientation Dependence of Magnetoresistance in Spin-Dependent Tunnel Junctions
}

\author{
Wen-C. Chiang, Y. M. Chang, C. H. Ho, Y. D. Yao, and Minn-Tsong Lin
}

\begin{abstract}
The dependence of magnetotransport on field orientation is an important issue in spintronics-related devices where the applied field is not necessarily in the ideal field-in-plane (FIP) geometry. In this study, we perform tunneling magnetoresistance (TMR) measurements on $\mathrm{Co}-\mathrm{Al}_{2} \mathrm{O}_{3}-\mathrm{CoFe}-\mathrm{NiFe}$ spin-dependent tunnel (SDT) junctions prepared at different conditions with varying field orientation ranging from FIP to field-perpendicular-to-plane (FPP). The TMR ratio decreases drastically, whereas the switching field of Co increases when the field direction is set close to FPP. Furthermore, in a situation near FPP, a peculiar TMR looping behavior is observed for one set of samples. Interface effect is thought to be related.
\end{abstract}

Index Terms-Field-in-plane (FIP), field-perpendicular-to-plane (FPP), spin-dependent tunnel (SDT) junction, tunneling magnetoresistance (TMR).

\section{INTRODUCTION}

$\mathbf{S}^{\mathrm{p}}$ PIN-DEPENDENT tunnel (SDT) junctions consisting of two ferromagnetic (FM) layers separated by a thin nonmagnetic tunneling barrier have been studied extensively in recent years for the interest that they exhibit large tunneling magnetoresistances (TMR) and, hence, are directly linked to technological applications [1]. Most TMR studies utilize the field-inplane (FIP) configuration in which the FM spin directions are manipulated along the geometric easy axis. In the FIP geometry, the tunneling conductance $G$ of the junction can be described as

$$
G=G_{\mathrm{fbf}^{\prime}}^{\prime}\left(1+P_{\mathrm{fb}} P_{\mathrm{f}^{\prime} \mathrm{b}} \cos \theta\right)
$$

where $P_{\mathrm{fb}}$ and $P_{\mathrm{f}^{\prime} \mathrm{b}}$ are the two effective ferromagnet-insulator coupled spin polarizations, $G_{\mathrm{fbf}^{\prime}}^{\prime}$ is a constant, and $\theta$ is the angle between the two FM spin directions [2]. The electron transport

Manuscript received August 11, 2004. The work of W.-C. Chiang was supported by the National Science Council of Taiwan under Grant NSC 92-2112-M034-001. The work of M.-T. Lin was supported by the National Science Council of Taiwan under Grant 92-2216-E-002-018 and by MOEA Nanostorage Project 92-EC-17-A-08-S1-0006.

W.-C. Chiang is with the Department of Physics, Chinese Culture University, 111 Taipei, Taiwan, R.O.C. (e-mail: wchiang @ faculty.pccu.edu.tw).

Y. M. Chang is with the Department of Physics, National Taiwan University, 106 Taipei, Taiwan, R.O.C. (e-mail: ymchang@ @hys.ntu.edu.tw).

C. H. Ho was with the Department of Physics, National Taiwan University, 106 Taipei, Taiwan, R.O.C. He is now with Macronix International Co., Ltd., 300 Hsinchu, Taiwan, R.O.C. (e-mail: chiahuaho@mxic.com.tw).

Y. D. Yao is with the Institute of Physics, Academia Sinica, 115 Taipei, Taiwan, R.O.C. (e-mail: phyao@gate.sinica.edu.tw).

M.-T. Lin is with the Department of Physics and Center for Nanostorage Research, National Taiwan University, 106 Taipei, Taiwan, R.O.C., and also with the Institute of Atomic and Molecular Sciences, Academia Sinica, 106 Taipei, Taiwan, R.O.C. (e-mail: mtlin@phys.ntu.edu.tw).

Digital Object Identifier 10.1109/TMAG.2004.842082 of the junction through the barrier is spin-dependent and modulated by the relative orientation of the two FM spins.

In contrast to FIP, the FM layers within the SDT junction in the field-perpendicular-to-plane (FPP) geometry often demand a larger field to align their moments in the hard-axis direction due to the geometric anisotropy, leading to the prolongation of the MR saturation [3]. The electronic structure of the FM layers in the sputtered junction is often assumed identical regardless of lateral or vertical orientation, but the switching of the FM moments complicates when the field is set away from the in-plane direction. Interfacial coupling starts to play a more crucial role. The localized magnetic domains formed via dipole interactions are found to be very sensitive to interfacial roughness and the way external field is applied [4], [5]. The antiparallel (AP) state of the two bulk FM layers, which gives rise to the tunneling resistance and dominates in the FIP geometry, might not be a stable state in the FPP situation [6]. In the presence of uniaxial anisotropy (not necessarily along the geometric easy axis), a perpendicular field contribution is likely even in the perfect FIP situation. A relevant issue is found in the magnetoresistive random-access memory (MRAM) design where the threshold for bit switching depends strongly on anisotropy and the combination of easy and hard direction fields. Thus, the study of TMR with changing field orientation is of both fundamental and technological importance.

Here, we demonstrate our study of TMR with varying field orientation on $\mathrm{Co}-\mathrm{Al}_{2} \mathrm{O}_{3}-\mathrm{CoFe}-\mathrm{NiFe}$ pseudospin-valve-type SDT junctions prepared at different conditions. The TMR ratio is analyzed along various field angles and is compared for different types of samples. In one type of samples in which the $\mathrm{Al}$ oxidation process is altered, an anomalous FPP looping behavior in the TMR curve is observed and discussed.

\section{EXPERIMENT AND RESULTS}

$\left[\mathrm{Co}(10 \mathrm{~nm})-\mathrm{Al}_{2} \mathrm{O}_{3}(2.6)-\mathrm{CoFe}(1.2)-\mathrm{NiFe}(10)\right]$ SDT junctions were deposited onto glass substrates in a magnetron sputtering system having a base pressure of $\sim 1 \times 10^{-8}$ torr. The sample preparation details were stated elsewhere [7]. Co and $\mathrm{CoFe}-\mathrm{NiFe}$ were chosen for the pseudospin-valve-type FM elements because of their coercivity difference. Two series of samples were fabricated-series a made with our typical deposition conditions [7] and series $b$ made with an intentional exposure to air prior to the deposition of the top FM layer in order to investigate the impact of interfacial quality on the angular field dependence of MR. The TMR measurement was achieved by the standard four-probe technique at room temperature in the current-perpendicular-to-plane (CPP) geometry. 


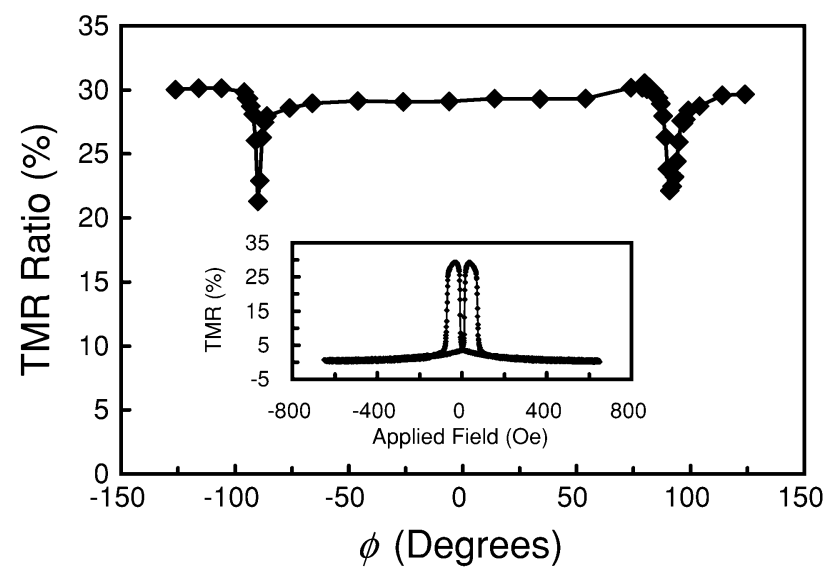

Fig. 1. TMR ratio versus $\phi$ for sample $a$, a typical $\mathrm{Co}-\mathrm{Al}_{2} \mathrm{O}_{3}-\mathrm{CoFe}-\mathrm{NiFe}$ SDT junction. The inset indicates its FIP TMR loop.

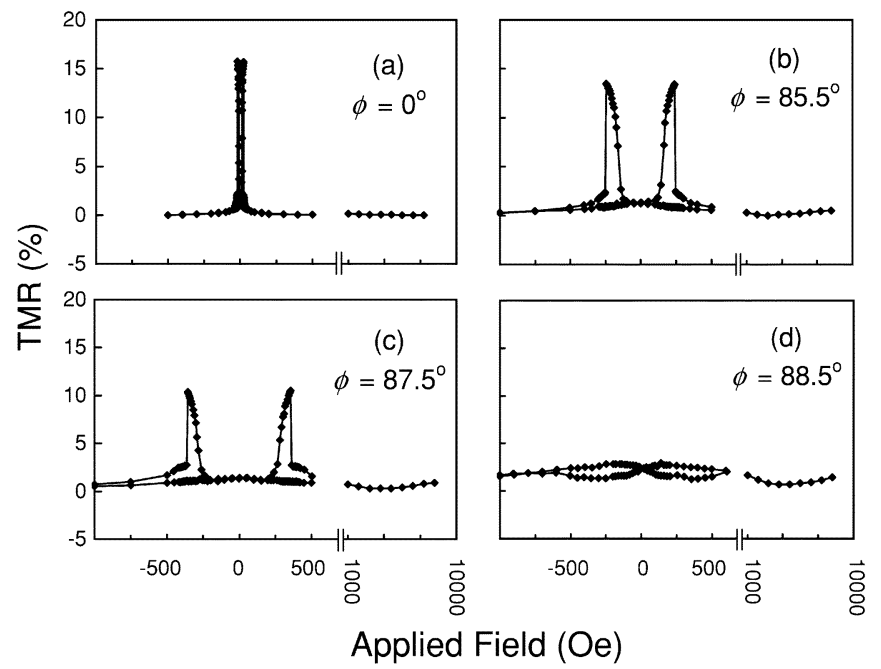

Fig. 2. TMR loops of sample $b$ for $\phi=$ (a) $0^{\circ}$, (b) $85.5^{\circ}$, (c) $87.5^{\circ}$, and (d) $88.5^{\circ}$. The positive end of each graph extends from 1 to $10 \mathrm{KOe}$.

A precise rotator of $0.2^{\circ}$ resolution was used to study the $\phi$ dependence of TMR, where $\phi$ is the angle between the applied field and the film plane. Sixteen samples within each series were measured identical or nearly identical. Thus, we choose two samples, sample $a$ and sample $b$ from series $a$ and series $b$, respectively, for the following presentation.

Fig. 1 shows the TMR ratio as a function of $\phi$, and the inset indicates the FIP TMR loop, plotted in the percentage change of TMR (with respect to the saturation value) versus applied field, for sample $a$. The "TMR ratio" is defined as the difference between the maximum and the saturated (minimum) resistance divided by the saturated resistance. Sample $a$ exhibits a 30\% in-plane TMR ratio. The TMR ratio remains roughly the same for most field orientations except for a small region where the configuration is close to FPP. The TMR ratio drops by a margin of $30 \%$ at $\phi \sim 90^{\circ}$.

Fig. 2 shows the TMR loops of sample $b$ for selected values of $\phi$. The in-plane TMR ratio is now only $\sim 15 \%$, indicating that its interfaces are different from those of sample $a$. All loops show a sloped rise and a steep descent corresponding to the switching of the $\mathrm{CoFe}-\mathrm{NiFe}$ and the Co moments, respectively. The field where the sharp descent occurs is in agreement with

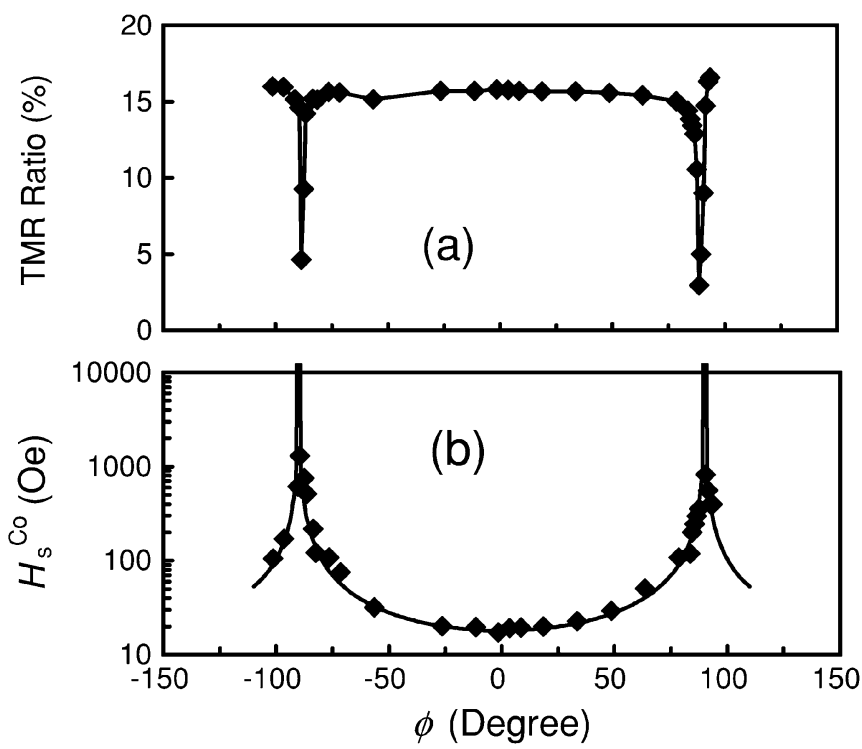

Fig. 3. (a) TMR ratio, and (b) the switching field of $\mathrm{Co}\left(\mathrm{H}_{\mathrm{S}}^{\mathrm{Co}}\right)$ as functions of $\phi$ for sample $b$. The solid line in (b) is the fitted result utilizing the inverse of $\cos \phi$.

the switching field $\mathrm{Co}\left(\mathrm{H}_{\mathrm{s}}^{\mathrm{Co}}\right)$, obtained from the magnetic hysteresis loop, where the Co moment reverses its direction almost instantaneously. High-field TMR behavior was also measured with $\phi$ variation and was included in each graph. Fig. 2(a) shows the typical SDT characteristic that the MR approaches saturation and remains unchanged as the field increases. However, in the neighborhood of FPP, as seen in Figs. 2(b)-(d), the MR curves do not flatten like normal saturation but increase with field beyond a minimum. This tailing-up behavior shows a strong dependence on $\phi$, and the trend is most prominent when the field is applied almost perpendicularly. The high-field curves of sample $a$, on the other hand, remain flat for all values of $\phi$.

Fig. 3 shows the TMR ratio and the switching field of Co $\left(\mathrm{H}_{\mathrm{s}}^{\mathrm{Co}}\right)$, each as a function of $\phi$ for samples $b$. The TMR ratio stays approximately the same $(\sim 15 \%)$ for $\phi$ less than $80^{\circ}$. With configuration being close to FPP, the TMR ratio decreases rapidly and reaches the lowest value $(\sim 4 \%)$ as $\phi$ is set almost equal to $90^{\circ}$. The drop of the TMR ratio is beyond $70 \%$-more drastic than that of sample $a(\sim 30 \%)$. The graphs also indicate that there should be a correspondence of the maximum value of $\mathrm{H}_{\mathrm{s}}^{\mathrm{Co}}$ with the lowest TMR ratio at the same $\phi$ close to the FPP geometry. A trigonometric fit to the $\mathrm{H}_{\mathrm{s}}^{\mathrm{Co}}(\phi)$ data exhibits a $|1 / \cos \phi|$ proportionality, and is displayed by the solid curve in Fig. 3(b). This proportionality and its symmetry about $\phi=0^{\circ}$ evidences that the magnetic easy axis is along the film plane in our sample.

Fig. 4 shows the magnified TMR loop of sample $b$, plotted in $R A$ (the product of the resistance $R$ and the junction's crosssectional area $A$ ) versus applied field, with $\phi=88.5^{\circ}$ and a field range of \pm 1500 Oe. In spite that the change is small, the $R A$ variation in the low-field region is anomalous in contrast to its regular behavior. When the field is ramped down from high fields, $R A$ increases and reaches its summit before the external field is set to zero. $R A$ then decreases, passing the zero-field point and climbing back up again before reaching the second height. The arrows in Fig. 4 indicate the looping sequences. The 


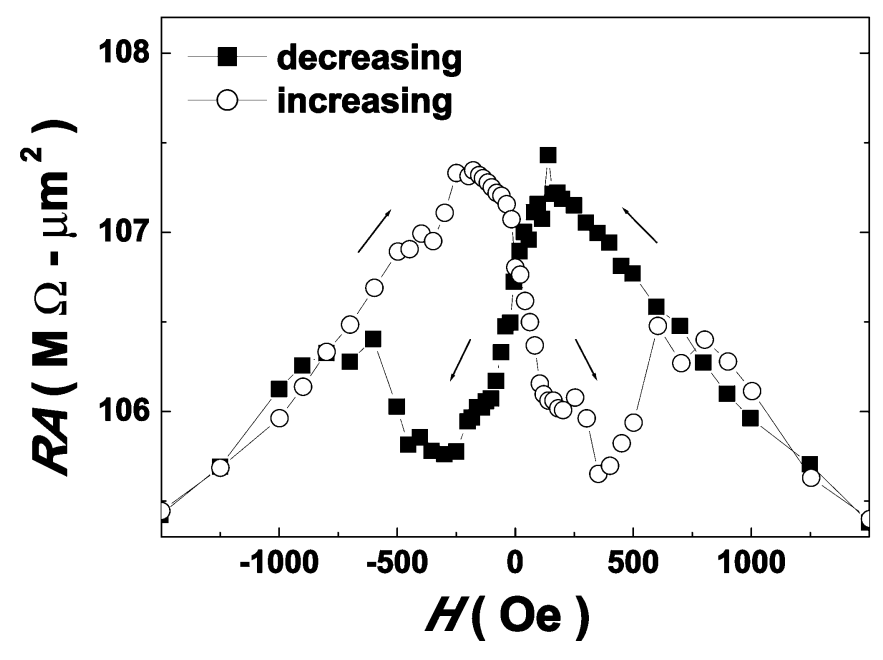

Fig. 4. TMR loop (plotted in $R A$ versus applied field $H$ ) for sample $b$ in the low-field region at $\phi=88.5^{\circ}$. The solid (open) symbols represent the data taken along the decreasing (increasing) field branch.

increasing and the decreasing field branches exhibit symmetric results, and the cycling is repeatable. This behavior is neither seen in other field angles nor in any configuration measured for sample $a$.

\section{DISCUSSION}

The peculiar $R A$ behavior shown in Fig. 4 seems analogous to the inverse giant magnetoresistance (GMR) found in $\mathrm{FeCrFe}-\mathrm{Cu}-\mathrm{Fe}-\mathrm{Cu}$ multilayers with imperfect FM coupling across $\mathrm{Cr}$ [8]. However, electron scattering plays a less important role in our SDT junctions, and thus the inverse spin asymmetry can hardly be responsible for the anomalous $R A$ variation. The sample's magnetic hysteresis loop confirms the typical behavior in which the most random spin state occurs after passing the zero-field point, implying that the anomaly is not originated from the bulk magnetization. Given that this anomaly is not seen in sample $a$, it becomes intuitive to presume that the cause is interface related. In the presence of interfacial roughness, as indicated in our previous work [7], the additional coupling of the dipole-dipole interaction cannot be ignored [9]. In the FPP geometry, the AP state of the two ferromagnets is suppressed, leading to the drastic decrease of the magnetoresistance; but on the other hand, a meta-stable AP state imposed by the interfacial magnetic structures could exist in the low-field region and result in the observed anomaly. The fact that both the low-field anomaly and the high-field tailing-up (see Fig. 2) are most prominent at $\phi=88.5^{\circ}$ indicates the nature of their causes could be related; and they both occur upon a point when the interfacial effect is most likely to dominate. To elucidate the origin, microscopic probing of localized interfacial domains is demanded. Future investigation, including the employment of more sensitive probing techniques such as neutron scattering, is under consideration.

\section{CONCLUSION}

We have shown that in our pseudospin-valve-type SDT junctions the TMR ratio drastically decreases upon setting the field close to the off-plane direction. This effect is much more significant for the junction with degraded interface quality (sample $b$ ) than for the typical one (sample $a$ ). Furthermore, for sample $b$, the TMR curve taken in the nearly FPP geometry shows a peculiar behavior in the small-field region in which the maximum value of $R A$ rises along looping before reaching the zero-field point. At high fields, its value of $R A$ does not remain saturated but tails up with increasing field. The results suggest that interfacial quality plays a key role for magnetotransport and the contribution by the off-plane field is substantial. Those factors should be taken into account in future applications when complicated field distribution is involved.

\section{REFERENCES}

[1] J. S. Moodera, L. R. Kinder, T. M. Wong, and R. Meservey, "Large magnetoresistance at room temperature in ferromagnetic thin film tunnel junctions," Phys. Rev. Lett., vol. 74, pp. 3273-3276, Apr. 1995.

[2] J. C. Slonczewski, "Conductance and exchange coupling of two ferromagnets separated by a tunneling barrier," Phys. Rev. B, Condens. Matter, vol. 39, pp. 6995-7002, Apr. 1989.

[3] Y. Otani, T. Kohda, V. Novosad, K. Fukamichi, S. Yuasa, and T. Katayama, "Shape induced in-plane magnetic anisotropy reorientation in epitaxial hexagonal close packed cobalt dots," J. Appl. Phys., vol. 87, pp. 5621-5623, May 2000.

[4] J. Rothman, M. Klaui, L. Lopez-Diaz, C. A. F. Vaz, A. Bleloch, J. A. C. Bland, Z. Cui, and R. Speaks, "Observation of a Bi-domain state and nucleation free switching in mesoscopic ring magnets," Phys. Rev. Lett., vol. 86, pp. 1098-1101, Feb. 2001.

[5] S. P. Li, W. S. Lew, J. A. C. Bland, M. Natali, A. Lebib, and Y. Chen, "Intrinsic anisotropy-defined magnetization reversal in submicron ring magnets," J. Appl. Phys., vol. 92, pp. 7397-7403, Dec. 2002.

[6] S. W. Yuan and H. N. Bettram, "Magnetic thin film domain wall motion under dynamic fields," J. Appl. Phys., vol. 72, pp. 1033-1038, Aug. 1992.

[7] M.-T. Lin, C. H. Ho, Y. D. Yao, R. T. Huang, C. C. Liao, F. R. Chen, and J. J. Kai, "Interface characterization and thermal stability of Co/AlO/CoFe spin-dependent tunnel junctions," J. Appl. Phys., vol. 91, pp. 7475-7477, May 2002.

[8] J. M. George, L. G. Pereira, A. Barthélémy, F. Petroff, L. Steren, J. L. Duvail, A. Fert, R. Loloee, P. Holody, and P. A. Schroeder, "Inverse spin-valve-type magnetoresistance in spin engineered multilayered structures," Phys. Rev. Lett., vol. 72, pp. 408-412, Jan. 1994.

[9] D. C. Parks, P. J. Chen, W. F. Egelhoff Jr., and R. D. Gomez, "Interfacial roughness effects on interlayer coupling in spin valves grown on different seed layers," J. Appl. Phys., vol. 87, pp. 3023-3026, Mar. 2000. 